Psychotherapeut $2021 \cdot 66: 511-517$ https://doi.org/10.1007/s00278-021-00523-4 Angenommen: 3. Juni 2021 Online publiziert: 7. Juli 2021

๑) Der/die Autor(en) 2021

\section{Geschlechtsspezifische Unterschiede in der psychotherapeutischen Versorgung}

\author{
Aktuelle Daten aus Österreich
}

\author{
Friedrich Riffer ${ }^{1,2} \cdot$ Magdalena Knopp ${ }^{3} \cdot$ Juliane Burghardt ${ }^{3}$ Manuel Sprung ${ }^{1,2,3}$ \\ ' Universitätsklinikum für Psychosomatische Medizin Eggenburg, Psychosomatisches Zentrum \\ Waldviertel, Eggenburg, Österreich \\ ${ }^{2}$ Rehabilitationsklinik Gars am Kamp, Psychosomatisches Zentrum Waldviertel, Gars am Kamp, Österreich \\ ${ }^{3}$ Department für Psychologie und Psychodynamik, Fachbereich für Klinische Psychologie, Karl Landsteiner \\ Privatuniversität für Gesundheitswissenschaften, Krems, Österreich
}

Hintergrund: Geschlechtsspezifische Unterschiede bei psychischen Erkrankungen sind vielfach belegt. Auch bei der Inanspruchnahme psychotherapeutischer und medizinischer Versorgungsangebote sind Geschlechtsunterschiede festzustellen. Die vorliegende Studie untersucht Geschlechtsunterschiede bei der Inanspruchnahme psychotherapeutischer Versorgungsangebote in Österreich, da hierzu bisher keine Studien vorliegen.

Material und Methoden: Eine Stichprobe von 1909 Patient*innen (64\% Frauen) mit einer psychischen Erkrankung wurde im Rahmen einer stationären Behandlung in einer psychosomatischen Klinik in Österreich zur Inanspruchnahme ambulanter psychotherapeutischer Behandlung befragt. Sowohl psychotherapeutische Vorbehandlung als auch geplante weiterführende Behandlung wurden einbezogen. Ergebnisse: Der größte Teil der Patient*innen (70\%) litt seit mehr als 2 Jahren an einer psychischen Erkrankung, und knapp die Hälfte (45\%) war bereits zuvor in stationärer und $82 \%$ in ambulanter psychotherapeutischer Behandlung. Demnach handelt es sich um eine Stichprobe chronisch psychisch kranker Menschen. Die Studienergebnisse zeigten geschlechtsspezifische Unterschiede bei der Inanspruchnahme psychotherapeutischer Behandlung. Männer gingen seltener zum Arzt, waren weniger häufig in psychotherapeutischer Vorbehandlung (Männer: 79\%; Frauen: $84 \%$ ) und planten weniger häufig eine weiterführende Behandlung im Anschluss an eine stationäre Behandlung als Frauen.

Schlussfolgerung: Die Geschlechtsunterschiede bei der Inanspruchnahme von psychotherapeutischer Behandlung könnten damit erklärt werden, dass es Unterschiede in den gesellschaftlichen Erwartungen gegenüber Männern und Frauen gibt. Die Behandlungsmotivation von Männern könnte durch eine geschlechtsspezifische Behandlungsstrategie gefördert werden.

\section{Schlüsselwörter}

Psychische Erkrankungen · Ambulante Versorgung · Männer · Frauen · Österreich
Ambulante Psychotherapie wird zu zwei Dritteln von Frauen und zu einem Drittel von Männern in Anspruch genommen. Diese Unterschiede der Inanspruchnahme setzen sich in anderen Bereichen des Gesundheitswesens fort. Die Vermutung liegt nahe, dass dies in der erhöhten Symptombelastung von Frauen begründet ist. Die Literatur zeigt allerdings, dass Inanspruchnahmeverhalten und Symptombelastung relativ unabhängig voneinander und Geschlechterunterschiede 
selbst dann zu finden sind, wenn sich die Symptombelastung von Männern und Frauen annähert. Die vorliegende Studie untersucht Geschlechtsunterschiede bei der Inanspruchnahme psychotherapeutischer Versorgungsangebote in Österreich und diskutiert diesbezügliche Ursachen und potenziell zu ziehende Konsequenzen in der Therapie.

\section{Einleitung}

Es ist vielfach belegt, dass es teils deutliche Geschlechtsunterschiede bei der Häufigkeit psychischer Erkrankungen gibt (Wittchen und Hoyer 2011). Frauen leiden häufiger an Essstörungen, Depressionen und Panikstörungen; wohingegen Männer häufiger Suchterkrankungen und Persönlichkeitsstörungen aufweisen. Geschlechtsunterschiede zeigen sich auch in der Inanspruchnahme von Psychotherapie (Riecher-Rössler 2008). Ambulante Psychotherapie wird zu zwei Dritteln von Frauen und zu einem Drittel von Männern in Anspruch genommen (Strauß et al. 2002). Diese Unterschiede setzen sich in anderen Bereichen des Gesundheitswesens fort. So nehmen Männer weniger häufig medizinische Behandlungen in Anspruch als Frauen (Ladwig et al. 2000; Bertakis et al. 2000). Die Vermutung liegt nahe, dass die erhöhte Symptombelastung von Frauen zur höheren Inanspruchnahme führt. Die Literatur zeigt allerdings, dass diese beiden Faktoren relativ unabhängig voneinander sind und die Geschlechterunterschiede auch zu finden sind, wenn sich die Symptombelastung von Männern und Frauen annähert (Ladwig et al. 2000). Stattdessen wird vermutet, dass Unterschiede in den gesellschaftlichen Erwartungen gegenüber Männern und Frauen die Unterschiede in der Inanspruchnahme erklären. Die typischen geschlechtsspezifischen Erwartungen erschweren es Männern, Bedenken und Sorgen um ihre Gesundheit auszudrücken (Yousaf et al. 2015a). Dies behindert ihre Fähigkeit, professionelle psychologische Hilfe in Anspruch zu nehmen (Hammer und Vogel 2010).

Nicht nur die Mehrheit der Patient ${ }^{*}$ innen ist weiblich, auch die Mehrheit der Psychotherapeut*innen sind Frauen (Schigl 2019). Fast drei Viertel aller Psychotherapeut*innen sind weiblich. Das bedeutet, dass in der Praxis am häufigsten Frauen von Frauen behandelt werden.

Die Besonderheiten der österreichischen Psychotherapieversorgung und -ausbildung lassen offen, ob die bisherigen Studienergebnisse auch in Deutschland anwendbar sind. Nur ein sehr geringer Teil der psychotherapeutischen Behandlung in niedergelassener Praxis wird vollständig durch die Krankenkasse finanziert (LöfflerStastka und Hochgerner 2021). Der größte Teil der Patient*innen muss die ambulante Psychotherapie bis auf einen Kostenzuschuss von $28 €$ selbst bezahlen. Die Ausbildung zum/zur Psychotherapeut*in ist in Österreich, wie zukünftig auch in Deutschland, eine Ausbildung, die ohne Psychologiestudium zugänglich ist (Datler et al. 2021). Bisher wurden keine Studien zu geschlechtsspezifischen Unterschieden in der psychotherapeutischen Versorgung in Österreich veröffentlicht. Die vorliegende Studie untersucht geschlechtsspezifische Unterschiede bei der Inanspruchnahme von ambulanter Psychotherapie anhand einer großen Stichprobe psychisch kranker Menschen in stationärer Behandlung.

\section{Material und Methoden}

\section{Stichprobe}

Eine Stichprobe mit 1909 Patient*innen, 1224 (64\%) Frauen und 685 (36\%) Männer, wurde über ihre psychotherapeutische Vorbehandlung sowie ihre geplante weiterführende Behandlung befragt. Die Patient*innen befanden sich aufgrund einer psychischen Erkrankung in stationärer Behandlung in einer psychosomatischen Klinik in Österreich. Das durchschnittliche Alter der Patient*innen betrug 45,5 Jahre (SD $\pm 11,9$ Jahre). Frauen waren im Durchschnitt 44,8 Jahre (SD \pm 11,9 Jahre) alt, wohingegen Männer 46,6 Jahre (SD $\pm 11,3$ Jahre) alt waren.

Das Psychosomatische Zentrum Waldviertel (PSZW) umfasst das Universitätsklinikum für Psychosomatische Medizin Eggenburg und die psychiatrische Rehabilitationsklinik Gars am Kamp. Die aktuelle Stichprobe setzt sich aus 1064 Patient*innen aus dem Universitätsklinikum Eggenburg und 845 aus der Rehabilitationsklinik Gars am Kamp zusammen. In der Klinik Eggenburg wer- den v. a.Patient*innen mit Persönlichkeits- und Verhaltensstörungen (ICD-10 $\mathrm{F} 60-\mathrm{F} 69=12,6 \%$ ), affektiven Störungen (ICD-10 F30-F39=22,3\%), psychischen und Verhaltensstörungen durch Alkohol (ICD-10 F10 =12,8\%), neurotischen, Belastungs- und somatoformen Störungen (ICD-10 F40-F48=34\%), Essstörungen (ICD-10 F50 =15,8\%) und verschiedenen anderen Störungsbildern 2,5\% behandelt. In der Rehabilitationsklinik Gars am Kamp werden überwiegend Menschen mit affektiven Störungen (ICD-10 $\mathrm{F} 30-\mathrm{F} 39=63,6 \%)$ sowie neurotischen, Belastungs- und somatoformen Störungen (ICD-10 F40-F48=28,3\%) therapiert (Riffer et al. 2017, 2018; Sprung et al. 2021).

\section{Setting}

Die Daten des PSZW wurden zwischen Juli 2017 und Juli 2018 erhoben. Das Universitätsklinikum Eggenburg ist eine wichtige Anlaufstelle für chronisch psychisch kranke Menschen und hält 100 stationäre Betten vor. Die interdisziplinäre Behandlung erfolgt in Kompetenzbereichen, die nach unterschiedlichen Störungsbildern gegliedert sind. In den Kompetenzbereichen werden Menschen mit Traumafolgeerkrankungen, Persönlichkeitsstörungen, affektiven, Angst- und Zwangsstörungen, Sucht und Abhängigkeitserkrankungen, chronischen Schmerzerkrankungen sowie Essstörungen behandelt (Riffer et al. 2016). Der stationäre Aufenthalt im Universitätsklinikum Eggenburg umfasst 8 bis 12 Wochen, in denen die Patient ${ }^{*}$ innen eine intensive und störungsspezifische Therapie erhalten. Einschlusskriterium der Studie ist die Aufnahme im PSZW im oben angeführten Zeitraum. Aufgrund des vielfältigen Therapieangebots ist die Therapiefähigkeit der Patient*innen entscheidend für die Aufnahme. Ausschlusskriterien sind akute Psychosen, suizidales Verhalten und Patient*innen mit akuter Intoxikation. In der psychiatrischen Rehabilitationsklinik Gars am Kamp beträgt die Aufenthaltsdauer in der Mehrheit der Fälle 6 Wochen. Die ebenfalls interdisziplinäre Behandlung ist störungsunspezifisch. 
Tab. 1 Dauer der Arbeitsunfähigkeit und Haushaltseinkommen bei Klinikaufnahme, differenziert nach dem Geschlecht

\begin{tabular}{|c|c|c|c|c|}
\hline $\begin{array}{l}\text { Sozioökonomische } \\
\text { Daten }\end{array}$ & $\begin{array}{l}\text { Frauen } \\
\text { (Anteil \% [Anzahl n]) }\end{array}$ & $\begin{array}{l}\text { Männer } \\
\text { (Anteil \% [Anzahl n]) }\end{array}$ & $x^{2}(d f)$ & $p$ \\
\hline \multicolumn{5}{|c|}{ Dauer der Arbeitsunfähigkeit ${ }^{a}$} \\
\hline Bis 5 Woche (1 Mo.) & $26,0(190)$ & $29,5(126)$ & \multirow{4}{*}{$\begin{array}{l}3,8 \\
(3)\end{array}$} & \multirow[t]{4}{*}{0,276} \\
\hline Bis 15 Wochen (3 Mo.) & $20,7(151)$ & $19,9(85)$ & & \\
\hline Bis 30 Wochen (6 Mo.) & $14,1(103)$ & $16,2(69)$ & & \\
\hline $\begin{array}{l}\text { Mehr als } 30 \text { Wochen } \\
\text { (>6 Mo.) }\end{array}$ & $39,3(287)$ & $34,4(147)$ & & \\
\hline \multicolumn{5}{|c|}{ Haushaltseinkommen (netto/monatlich) } \\
\hline Weniger als $€ 1000$ & $33,2(264)$ & $23,0(105)$ & \multirow[t]{3}{*}{$27,7(2)$} & \multirow[t]{3}{*}{0,001} \\
\hline$€ 1000-2000$ & $42,7(339)$ & $41,2(188)$ & & \\
\hline Mehr als $€ 2000$ & $24,1(191)$ & $35,7(163)$ & & \\
\hline
\end{tabular}

Tab. 2 Vor der Klinikaufnahme in Anspruch genommene psychotherapeutische Behandlung, differenziert nach dem Geschlecht

\begin{tabular}{|c|c|c|c|c|}
\hline $\begin{array}{l}\text { Behandlungs- } \\
\text { bezogene Daten }\end{array}$ & $\begin{array}{l}\text { Frauen } \\
\text { (Anteil \% [Anzahl } n] \text { ) }\end{array}$ & $\begin{array}{l}\text { Männer } \\
\text { (Anteil \% [Anzahl } n] \text { ) }\end{array}$ & $x^{2}(d f)$ & $p$ \\
\hline \multicolumn{5}{|c|}{ Psychotherapeutische Vorbehandlung (ambulant) } \\
\hline $\mathrm{Ja}$ & $84,2(702)$ & $78,6(372)$ & \multirow[t]{2}{*}{$7,4(1)$} & \multirow[t]{2}{*}{0,006} \\
\hline Nein & $15,8(132)$ & $21,4(101)$ & & \\
\hline \multicolumn{5}{|c|}{ Kassenplatz (kostenlose Behandlung) } \\
\hline $\mathrm{Ja}$ & $43,4(302)$ & $44,8(165)$ & \multirow[t]{2}{*}{$0,08(1)$} & \multirow[t]{2}{*}{0,777} \\
\hline Nein & $56,6(394)$ & $55,2(203)$ & & \\
\hline \multicolumn{5}{|c|}{ Kosten für nichtkassenfinanzierte Psychotherapie (je Einheit/h) } \\
\hline Bis $€ 50$ & $15,8(61)$ & $15,7(31)$ & \multirow[t]{3}{*}{$1,4(2)$} & \multirow[t]{3}{*}{0,495} \\
\hline Bis $€ 100$ & $73,4(284)$ & $70,2(139)$ & & \\
\hline Mehr als $€ 100$ & $10,9(42)$ & $14,1(28)$ & & \\
\hline \multicolumn{5}{|c|}{ Wartezeit auf Kassenplatz } \\
\hline Bis 1 Woche & $5,9(50)$ & $8,4(40)$ & \multirow[t]{5}{*}{$6,0(4)$} & \multirow[t]{5}{*}{0,203} \\
\hline Bis 5 Wochen (1 Mo.) & $13,3(112)$ & $13,3(63)$ & & \\
\hline $\begin{array}{l}\text { Bis } 15 \text { Wochen } \\
\text { (3 Mo.) }\end{array}$ & $7,7(65)$ & $5,9(28)$ & & \\
\hline $\begin{array}{l}\text { Bis } 30 \text { Wochen } \\
\text { (6 Mo.) }\end{array}$ & $2,3(19)$ & $1,9(9)$ & & \\
\hline $\begin{array}{l}\text { Mehr als } 30 \text { Wochen } \\
\text { (> } 6 \text { Mo.) }\end{array}$ & $4,7(40)$ & $3,8(18)$ & & \\
\hline \multicolumn{5}{|c|}{ Gesamtdauer der psychotherapeutischen Vorbehandlung (ambulant) } \\
\hline Bis 3 Monate & $17,7(122)$ & $30,5(112)$ & \multirow[t]{3}{*}{$24,3(2)$} & \multirow[t]{3}{*}{0,001} \\
\hline Bis $1 \mathrm{Jahr}$ & $23,8(164)$ & $22,9(84)$ & & \\
\hline Länger als 1 Jahr & $58,6(404)$ & $46,6(171)$ & & \\
\hline \multicolumn{5}{|c|}{ Psychotherapeutische Behandlungen (ambulant) in den letzten 12 Monaten } \\
\hline 1 - bis 5 -mal & $21,6(143)$ & $33,4(114)$ & \multirow[t]{4}{*}{$24,7(3)$} & \multirow[t]{4}{*}{0,001} \\
\hline 6- bis 10-mal & $20,4(135)$ & $22,3(76)$ & & \\
\hline 11- bis 20-mal & $23,8(157)$ & $21,7(74)$ & & \\
\hline Mehr als 20-mal & $34,2(226)$ & $22,6(77)$ & & \\
\hline
\end{tabular}

Erhebungsverfahren

Im Rahmen der routinemäßigen Evaluation wurden zu Beginn und gegen Ende des stationären Aufenthalts soziodemografische und sozioökonomische Informationen, gesundheits- und krankheitsbezogene Informationen sowie Informationen zu psychotherapeutischer Vorbehandlung und geplanter weiterführender Behandlung, ermittelt. Die Daten wurden mithilfe eines computerbasierten Erhebungsverfahrens, dem Computer-based Health Evaluation System (Evaluation Software Development [ePRO, CHES], Innsbruck, Österreich; Holzner et al. 2012) erhoben und anschließend mithilfe eines Statistikprogramms (SPSS, Version 24.0) ausgewertet.

\section{Statistische Datenauswertung}

Soziodemografische und sozioökonomische Informationen sowie Angaben zu psychotherapeutischer Vorbehandlung und geplanter weiterführender Behandlung wurden in Bezug auf geschlechtsspezifische Unterschiede analysiert. Die Daten wurden deskriptiv ausgewertet und anschließend inferenzstatistisch mithilfe des $X^{2}$-Tests auf signifikante Unterschiede in den Häufigkeitsverteilungen geprüft. Bei einem $p$-Wert $<0,05$ unterscheidet sich das Antwortverhalten der Frauen von dem der Männer.

\section{Ergebnisse}

Sozioökonomische und krankheitsbezogene Daten

Die Ergebnisse der sozioökonomischen Datenauswertung bezüglich Arbeitsunfähigkeit und Haushaltseinkommen der Patient*innen bei Klinikaufnahme sind in - Tab. 1 jeweils im geschlechtsspezifischen Vergleich dargestellt. Zum Zeitpunkt der Aufnahme waren $71,5 \%$ der Patient*innen arbeitsunfähig. Etwas mehr als ein Drittel $(37,4 \%)$ gaben an, dass sie in den letzten 12 Monaten länger als 30 Wochen arbeitsunfähig waren.

Hinsichtlich der Nutzung des Gesundheitssystems zeigten sich geschlechtsspezifische Unterschiede. Mehr als ein Viertel der Frauen $(28,4 \%)$ verbrachten in den letzten 12 Monaten länger als 6 Wochen 


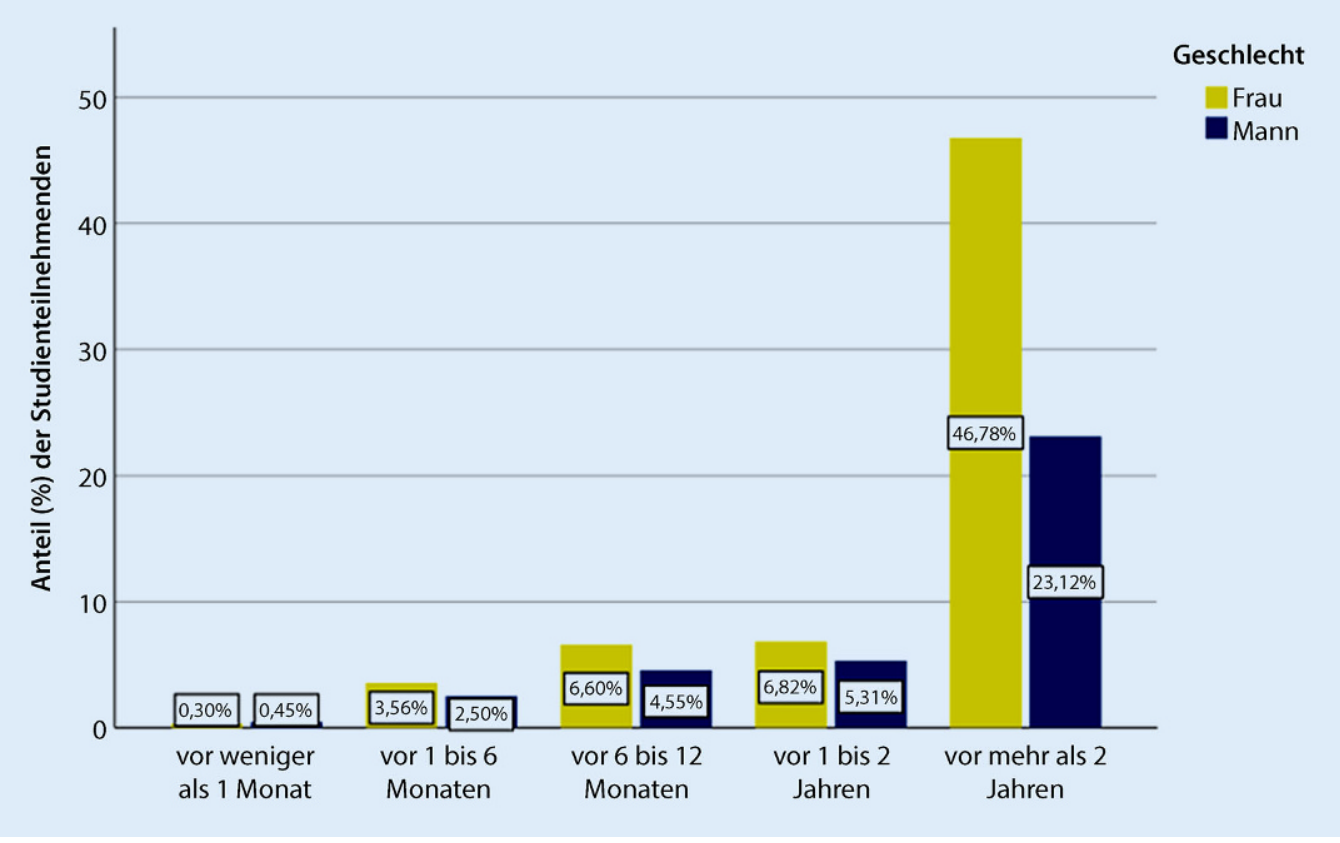

Abb. $1 \varangle$ Zeitpunkt des Erkrankungsbeginns vor der Klinikaufnahme im Vergleich zwischen Frauen und Männern

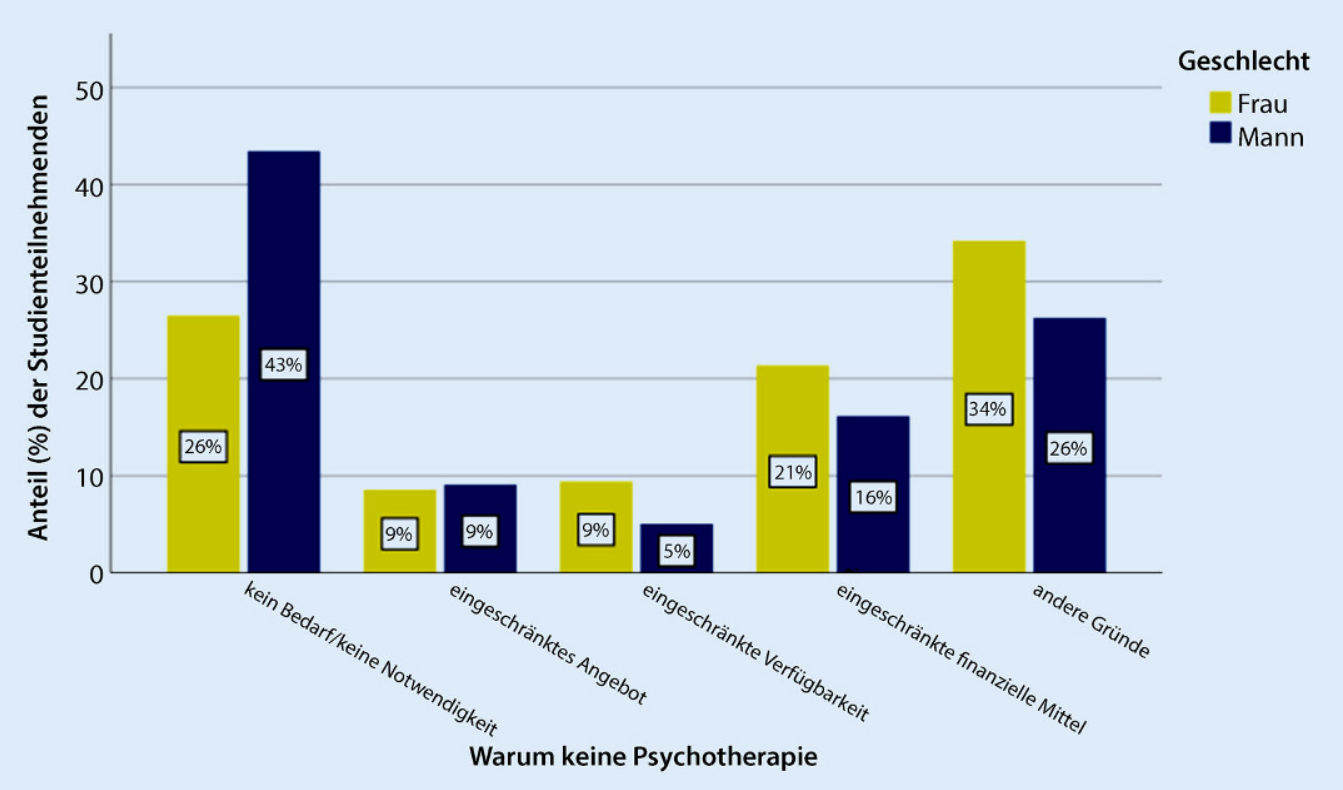

Abb. $2 \triangleleft$ Gründe, warum vor der Klinikaufnahme keine psychotherapeutische Vorbehandlung in Anspruch genommen wurde

zur stationären Behandlung im Krankenhaus, im Gegensatz zu 17,5\% der Männer. Männer gingen im Vergleich zu Frauen signifikant seltener zum Arzt. Bei den Frauen gingen $23,9 \%$ in den letzten 12 Monaten mehr als 20-mal zum Arzt. Dieser Anteil war bei Männern mit 17,2\% ebenfalls hoch, aber niedriger als bei den Frauen $\left(x^{2}=17,53 ; p \leq 0,001\right)$.

Bezogen auf das Haushaltseinkommen fand sich ebenfalls ein geschlechtsspezifischer Unterschied $\left(X^{2}=27,71 ; p<0,001\right)$. Der Anteil der Frauen mit einem Nied- rigsteinkommen $(<1000 €)$ war deutlich höher als der der Männer.

Mehr als die Hälfte (58\%) der Patient*innen waren demnach chronisch krank. Der größte Teil der Patient*innen litt seit mehr als 2 Jahren an einer psychischen Erkrankung (70\%). Angaben zum Beginn der Erkrankung finden sich in - Abb. 1. 
Tab. 3 Bei Klinikentlassung geplante psychotherapeutische Weiterbehandlung, differenziert nach dem Geschlecht

\begin{tabular}{|c|c|c|c|c|}
\hline $\begin{array}{l}\text { Behandlungsbezogene } \\
\text { Daten }\end{array}$ & $\begin{array}{l}\text { Frauen } \\
\text { (Anteil \% [An- } \\
\text { zahl } n] \text { ) }\end{array}$ & $\begin{array}{l}\text { Männer } \\
\text { (Anteil \% [An- } \\
\text { zahl } n] \text { ) }\end{array}$ & $x^{2}(d f)$ & $p$ \\
\hline \multicolumn{5}{|c|}{ Geplante psychotherapeutische Behandlung } \\
\hline Ja, stationär & $4,4(28)$ & $2,7(9)$ & \multirow[t]{3}{*}{$14,8(2)$} & \multirow[t]{3}{*}{0,001} \\
\hline Ja, ambulant & $90,5(578)$ & $85,4(287)$ & & \\
\hline $\begin{array}{l}\text { Keine weiterführende } \\
\text { Behandlung geplant }\end{array}$ & $5,2(33)$ & $11,9(40)$ & & \\
\hline \multicolumn{5}{|c|}{ Weiterbehandlungstermin bereits vereinbart } \\
\hline $\mathrm{Ja}$ & $55,0(333)$ & $55,1(163)$ & \multirow{2}{*}{$\begin{array}{l}0,104 \\
(1)\end{array}$} & \multirow[t]{2}{*}{0,747} \\
\hline Nein & $45,0(272)$ & $44,9(133)$ & & \\
\hline \multicolumn{5}{|c|}{ Gründe, warum keine weiterführende psychotherapeutisches Behandlung geplant ist } \\
\hline $\begin{array}{l}\text { Kein Bedarf/ } \\
\text { Notwendigkeit }\end{array}$ & $23,3(7)$ & $40,0(14)$ & \multirow[t]{4}{*}{7,6} & \multirow[t]{4}{*}{$0,103^{\mathrm{a}}$} \\
\hline $\begin{array}{l}\text { Kein oder eingeschränk- } \\
\text { tes Angebot }\end{array}$ & $3,3(1)$ & $8,6(3)$ & & \\
\hline $\begin{array}{l}\text { Kein oder eingeschränkte } \\
\text { finanzielle Mittel }\end{array}$ & $33,3(10)$ & $17,1(6)$ & & \\
\hline Andere Gründe & $36,7(11)$ & $25,7(9)$ & & \\
\hline
\end{tabular}

und $47 \%$ der Männer bereits länger als ein Jahr gedauert. Bei den Personen, die eine Psychotherapie in Anspruch genommen hatten, unterschieden sich zusätzlich die Behandlungsdauer $\left(X^{2}=24,31 ; p<0,001\right)$ und -intensität $\left(X^{2}=24,70 ; p<0,001\right)$ zwischen Männern und Frauen. Männer nahmen weniger Sitzungen in Anspruch, und die Therapie hatte eine geringere Gesamtdauer. Geschlechtsspezifische Unterschiede zeigten sich auch hinsichtlich der Gründe für bisher fehlende psychotherapeutische Behandlung (- Abb. 2). Deutlich mehr männliche (45\%) als weibliche (26\%) Patient*innen gaben an, keinen Bedarf bzw. keine Notwendigkeit für eine Behandlung gesehen zu haben. Frauen gaben häufiger als Männer an, dass sie keine Behandlung in Anspruch nahmen, weil ihnen die finanziellen Mittel fehlten.

Bei ihrer Entlassung gaben mehr als die Hälfte $(57 \%)$ der Patient*innen an, keinen Zugang zu einer kassenfinanzierten ambulanten Psychotherapie zu haben. Jene Patient*innen, die einen kassenfinanzierten ambulanten Psychotherapieplatz in Anspruch nahmen, warteten lange auf diesen: $40 \%$ bis zu 5 Wochen.

Die Gründe dafür, keine weiterführende psychotherapeutische Behandlung zu planen, wiesen ebenfalls Geschlechterunterschiede auf. Männer planten demnach seltener, eine weiterführende psychotherapeutische Behandlung in Anspruch zu nehmen, als Frauen $\left(X^{2}=14,75 ; p \leq 0,001\right)$. Die Ergebnisse sind 0 Tab. 3 zu entnehmen. Bei den Gründen gegen eine Weiterbehandlung berichteten Frauen häufiger als Männer, keine ausreichenden finanziellen Mittel zu haben.

\section{Diskussion}

Chronisch psychisch kranke Männer, die in einer Klinik in Österreich in stationärer psychosomatischer Behandlung waren, hatten vor der Behandlung weniger häufig psychotherapeutische und niedergelassene ärztliche Versorgungsangebote in Anspruch genommen als Frauen. Dies stimmt mit bisherigen Ergebnissen überein (Yousaf et al. 2015a). Im Besonderen hat sich gezeigt, dass Frauen sehr viel häufiger als Männer psychotherapeutische Behandlungen aufsuchen, obwohl sie nicht unbedingt häufiger psychisch krank sind (Ernst 2001). Als Ursache wird die traditionelle männliche Geschlechterrolle vermutet. Sie zeichnet sich dadurch aus, dass es Männern weniger als Frauen erlaubt ist, Schwächezu zeigen und mehr Unabhängigkeit verlangt wird. Dadurch wird es Männern erschwert, Sorge um ihre Gesundheit auszudrücken, was die Kommunikation mit dem Gesund- heitspersonal behindern kann (Yousaf et al. 2015a). In diesen Zusammenhang wird deutlich, dass es Männern, besonders solchen mit traditionellem Rollenverständnis, schwerer fällt, Hilfe in Anspruch zu nehmen (O'Neil 2008; Yousaf et al. 2015b). Dies ist auch aufgrund der häufigen komorbiden somatischen Erkrankungen sowie der erhöhten Mortalität von psychisch kranken Menschen besonders problematisch (Wittchen et al. 2011). Vermutlich trägt dieser Umstand dazu bei, dass Männer in Österreich durchschnittlich 5 Jahre kürzer leben als Frauen und eine dreifach höhere Suizidrate haben (Statistik Austria 2018). Darüber hinaus fanden Studien, dass es einen $\mathrm{Zu}$ sammenhang zwischen dem seltenen Hilfesuchverhalten von Männern und ihrer höherer Sterberate gibt (Hale et al. 2010).

Die Ergebnisse der vorliegenden Studie belegen, dass Männer auch ambulante psychotherapeutische Behandlung mit weniger Sitzungen und für eine kürzere Dauer in Anspruch nehmen als Frauen. Die ist möglicherweise ein Hinweis darauf, dass es bei Männern häufiger zu Therapieabbrüchen kommt als bei Frauen. Die Behandlungsmotivation von Männern könnte durch geschlechtsspezifische Behandlungsstrategie gefördert werden. Dazu gehört, dass Männer klar strukturierte Beratungsangebote bevorzugen (KrauseGirth 2004; Rudolf 2002), in denen sie an Entscheidungsprozessen beteiligt werden (Seidler et al. 2018). Folgende Gründe werden für eine mangelnde Inanspruchnahme psychosozialer und medizinischer Versorgungsangebote bei psychisch kranken Männern diskutiert: Scham hinsichtlich der Inanspruchnahme und das Bedürfnis nach emotionaler Kontrolle (Yousaf et al. 2015b). Verglichen mit Frauen, berichten Männer über mehr Schamerleben (Doherty und Kartalova-O'Doherty 2010).

Zudem zeigen die Ergebnisse der vorliegenden Studie, dass Frauen trotz geringerem Einkommen weniger kassenfinanzierte Plätze als Männer nutzen. Die Ergebnisse geben einen Hinweis darauf, dass besonders Frauen mit psychischen Erkrankungen vermehrt unter mangelnden finanziellen Mitteln leiden, sodass sie sich eine Psychotherapie nur leisten können, wenn sie kassenfinanziert ist. Um eine nachhaltige Stabilisierung der Verbesserung durch den stationären Krankenhaus- 
aufenthalt zu gewährleisten, ist eine ambulante Psychotherapie jedoch notwendig (Eichert 2016). Die Studienergebnisse spiegeln eine Ungleichheit in der Behandlung von psychischen und physischen Erkrankungen wider, da Behandlungen körperlicher Erkrankungen flächendeckend finanziert werden, psychischer Erkrankungen in Österreich allerdings nicht.

Die Daten beschränken sich auf Patient*innen in stationärer psychosomatischer Behandlung mit chronifizierten Verläufen. Zukünftige Studien sollten prüfen, ob sich die Ergebnisse auf weitere Patientengruppen übertragen lassen. Dennoch bestätigen die Ergebnisse anhand der großen Stichprobe Geschlechtsunterschiede bei der Inanspruchnahme psychotherapeutischer Behandlung.

\section{Fazit für die Praxis}

- Männer mit psychischen Erkrankungen nehmen weniger häufig eine psychotherapeutische Behandlung in Anspruch und gehen auch seltener zum Arzt als Frauen.

- Frauen mit psychischen Erkrankungen können eine notwendige psychotherapeutische Behandlung aufgrund fehlender finanzieller Mitteln teilweise nicht in Anspruch nehmen.

- Männer geben häufiger als Frauen an, keinen Bedarf/keine Notwendigkeit für eine Psychotherapie zu sehen.

Korrespondenzadresse

Magdalena Knopp, Msc.

Department für Psychologie und Psychodynamik, Fachbereich für Klinische Psychologie, Karl Landsteiner Privatuniversität für Gesundheitswissenschaften

Dr. Karl-Dorrek-Str. 30, 3500 Krems, Österreich Magdalena.knopp@kl.ac.at

Danksagungen. Wir bedanken uns bei der NÖ Forschungs- und Bildungsges. m.b.H. (NFB) für die finanzielle Unterstützung im Rahmen der Stiftungsprofessur für Klinische Psychologie. Des Weiteren bedanken wir uns für eine finanzielle Förderung durch das VAMED Institut für Gender Medizin und danken der Karl Landsteiner Privatuniversität für Gesundheitswissenschaften, Krems, Österreich, für ihre Unterstützung durch den Open Access Publikationsfonds.

\section{Gender-specific differences in psychotherapeutic care. Current data from Austria}

Background: Gender-specific differences in mental illnesses have been widely confirmed. Gender differences have also been found in the utilization of psychotherapeutic and medical care services. The present study is the first to examine gender differences in the utilization of psychotherapeutic care in Austria.

Material and methods: A sample of 1909 inpatients (64\% women) with a mental illness were questioned about the utilization of outpatient psychotherapeutic treatment during inpatient treatment at a psychosomatic clinic in Austria. Both outpatient psychotherapeutic treatment prior to the current inpatient psychosomatic treatment as well as the planned follow-up outpatient treatment were assessed.

Results: The majority of the patients $(70 \%)$ had been suffering from a mental disorder for more than 2 years, almost half ( $45 \%$ ) had already received inpatient treatment and $82 \%$ had already received outpatient treatment prior to the current treatment. Thus, the present data are from a sample of chronically mentally ill patients. The results showed gender-specific differences in the utilization of psychotherapeutic treatment. Men visited medical doctors less often than women, were less often in psychotherapeutic pretreatment (men 79\%, women $84 \%$ ) and also planned further follow-up inpatient treatment less often.

Conclusion: This gender difference in the utilization of psychotherapeutic treatment could be explained by the fact that there are differences in social expectations towards men and women. The motivation for treatment in men could be promoted by a genderspecific treatment strategy.

\section{Keywords}

Mental illness $\cdot$ Ambulatory care $\cdot$ Male $\cdot$ Female $\cdot$ Austria

\section{Einhaltung ethischer Richtlinien}

Interessenkonflikt. F. Riffer, M. Knopp, J. Burghardt und M. Sprung geben an, dass kein Interessenkonflikt besteht.

Die Vorgaben der gültigen Fassung der Deklaration von Helsinki wurden eingehalten. Ein positives Ethikvotum der Karl Landsteiner Privatuniversität liegt vor (EK-Nr: 1003/2021). Von allen an der Befragung Beteiligten liegt eine schriftliche Einverständniserklärung vor.

Open Access. Dieser Artikel wird unter der Creative Commons Namensnennung 4.0 International Lizenz veröffentlicht, welche die Nutzung, Vervielfältigung, Bearbeitung, Verbreitung und Wiedergabe in jeglichem Medium und Format erlaubt, sofern Sie den/die ursprünglichen Autor(en) und die Quelle ordnungsgemäßnennen, einen Link zur Creative Commons Lizenz beifügen und angeben, ob Änderungen vorgenommen wurden.

Die in diesem Artikel enthaltenen Bilder und sonstiges Drittmaterial unterliegen ebenfalls der genannten Creative Commons Lizenz, sofern sich aus der Abbildungslegende nichts anderes ergibt. Sofern das betreffende Material nicht unter der genannten Creative Commons Lizenz steht und die betreffende Handlung nicht nach gesetzlichen Vorschriften erlaubt ist, ist für die oben aufgeführten Weiterverwendungen des Materials die Einwilligung des jeweiligen Rechteinhabers einzuholen.
Weitere Details zur Lizenz entnehmen Sie bitte der Lizenzinformation auf http://creativecommons.org/ licenses/by/4.0/deed.de.

\section{Literatur}

Bertakis KD, Azari R, Helms LJ, Callahan EJ, Robbins JA (2000) Gender differences in the utilization of health care services. J Fam Pract 49:147-152

Datler W, Hochgerner M, Korunka C, Löffler-Stastka H, Pawlowsky G (2021) Disziplin, Profession und evidenzbasierte Praxis: Zur Stellung der Psychotherapie im Gesundheitssystem. Eine Bilanz. Psychother Forum. https://doi.org/10. 1007/s00729-021-00167-4

Doherty DT, Kartalova-O'Doherty Y (2010) Gender and self-reported mental health problems: predictors of help seeking from a general practitioner. Br J Health Psychol 15:213-228

Eichert H-C (2016in) 25. Rehabilitationswissenschaftliches Kolloquium. Deutscher Kongress für Rehabilitationsforschung; Gesundheitssystem im Wandel - Perspektiven der Rehabilitation, Aachen, 29. Februar bis 2. März 2016 Deutsche Rentenversicherung Bund, Berlin

Ernst C (2001) Die bessere und die schlechtere Hälfte? Geschlechtsunterschiede in der Prävalenz psychischer Krankheiten aus epidemiologischer Sicht. In: Riecher-Rössler A, Rohde A (Hrsg) Psychische Erkrankungen bei Frauen. Karger, , $S$ 47-61

Hale S, Grogan S, Willott S (2010) Male GPs' views on men seeking medical help: a qualitative study. $\mathrm{Br}$ JHealth Psychol 15:697-713 
Hammer JH, Vogel DL (2010) Men's help seeking for depression: the efficacy of a male-sensitive brochure about counseling. Couns Psychol 38:296-313

Holzner B, Giesinger JM, Pinggera J, Zugal $S$, Schöpf F, Oberguggenberger AS, Gamper EM Zabernigg A, Weber B, Rumpold G (2012) The Computer-based Health Evaluation Software (CHES): a software for electronic patientreported outcome monitoring. BMC Med Inform Decis Mak 12:126

Krause-Girth C (2004) Psychotherapie, Gesundheit und Geschlecht - Argumente für eine geschlechtersensible gesundheitsförderliche Psychotherapie. Psychother Forum 12:26-35

Ladwig K-H, Marten-Mittag B, Formanek B, Dammann G (2000) Gender differences of symptom reporting and medical health care utilization in the German population. Eur J Epidemiol 16:511-518

Löffler-Stastka H, Hochgerner M (2021) Versorgungswirksamkeit von Psychotherapie in Österreich. Psychoprax Neuroprax 24:57-61

O'Neil JM (2008) Summarizing 25 years of research on men's gender role conflict using the gender role conflict scale: new research paradigms and clinical implications. Couns Psychol 36:358-445

Riecher-Rössler A (2008) Geschlechtsspezifische Unterschiede in der Häufigkeit psychischer Störungen. Neurol Psychiatr 6(3):6-9

Riffer F, Kaiser E, Fellinger B (2016) Stationäre Therapie psychosomatischer Erkrankungen- ein Modell fürÖsterreich. JATROSNeurol Psychiatr 24:38-40

Riffer F, Sprung M, Kaiser E (2017) Evaluationsergebnisse der Rehabilitationsklinik Gars am Kamp. SpectrPsychiatr 3:34-37
Riffer F, Sprung M, Streibl L, Kaiser E (2018) Stationäre medizinische Rehabilitation von Patienten mit psychiatrischen oder psychosomatischen Erkrankungen: erste Evaluationsergebnisse der Rehabilitationsklinik Gars am Kamp. In Riffer F, Kaiser E, Sprung M, Streibl L (Hrsg) Das Fremde: Flucht - Trauma - Resilienz: Aktuelle traumaspezifische Konzepte in der Psychosomatik. Springer, Berlin, Heidelberg, S227-241

Rudolf G (2002) Gibt es nachweisbare Einflüsse der Geschlechtszugehörigkeit auf die Psychotherapie? In: Schweizer Charta für Psychotherapie (Hrsg) Mann oder Frau? Wie bestimmend ist das Geschlecht in der Psychotherapie? Edition Diskord, Tübingen, S75-95

Schigl B (2019) Gender als notwendige Perspektive psychotherapeutischen Handelns. Psychother Forum 23:18-24

Seidler ZE, Rice SM, Oliffe JL, Fogarty AS, Dhillon HM (2018) Men in and out of treatment for depression: strategies for improved engagement. Aust Psychol. https://doi.org/10.1111/ap.12331

Sprung M, Burghardt J, Streiblb L, Kaiser E, Jelemc H, Riffer F (2021) Effekte von Geschlecht und Alter auf die Ergebnisse von psychiatrisch/ psychosomatischer Rehabilitation. eingereicht

Statistik Austria (2018) Gender-Statistik. Statistik Austria, Wien

StraußB, Hartung J, Kächele H (2002) Geschlechterspezifische Inanspruchnahme von Psychotherapie und Soziale Arbeit. In: Hurrelmann K, Kolip P (Hrsg) Geschlecht, Gesundheit und Krankheit. Huber, Göttingen, S533-547
Wittchen H-U, Hoyer J (Hrsg) (2011) Klinische Psychologie \& Psychotherapie, 2. Aufl. Springer, Berlin Heidelberg

Wittchen HU, Jacobi F, Rehm J et al (2011) The size and burden of mental disorders and other disorders of the brain in Europe 2010. Eur Neuropsychopharmacol 21:655-679

Yousaf O, Grunfeld EA, Hunter MS (2015a) A systematic review of the factors associated with delays in medical and psychological help-seeking among men. Health Psychol Rev 9:264-276

Yousaf O, Popat A, Hunter MS (2015b) An investigation of masculinity attitudes, gender, and attitudes toward psychological help-seeking.Psychol Men Masculinity 16:234

\section{Hier steht eine Anzeige.}

\section{Springer}

